\title{
Relating genomic characteristics to environmental preferences and ubiquity in different microbial taxa
}

\author{
Marta Cobo-Simón and Javier Tamames
}

\begin{abstract}
Background: Despite the important role that microorganisms play in environmental processes, the low percentage of cultured microbes (5\%) has limited, until now, our knowledge of their ecological strategies. However, the development of high-throughput sequencing has generated a huge amount of genomic and metagenomic data without the need of culturing that can be used to study ecological questions. This study aims to estimate the functional capabilities, genomic sizes and 165 copy number of different taxa in relation to their ubiquity and their environmental preferences.

Results: To achieve this goal, we compiled data regarding the presence of each prokaryotic genera in diverse environments. Then, genomic characteristics such as genome size, $16 \mathrm{~S}$ rRNA gene copy number, and functional content of the genomes were related to their ubiquity and different environmental preferences of the corresponding taxa. The results showed clear correlations between genomic characteristics and environmental conditions.

Conclusions: Ubiquity and adaptation were linked to genome size, while $16 \mathrm{~S}$ copy number was not directly related to ubiquity. We observed that different combinations of these two characteristics delineate the different environments. Besides, the analysis of functional classes showed some clear signatures linked to particular environments.
\end{abstract}

Keywords: Microorganisms, High-throughput sequencing, Functional capabilities, Genomics, Co-occurrences

\section{Background}

Many microbes remain unculturable despite recent progress in cultivation techniques [1-7]. The development of high-throughput sequencing has provided a way to circumvent this limitation, producing a very large amount of genomic data that informs of the possible functions and capabilities of the targeted microorganisms. However, despite this huge amount of complex data [8], our knowledge of the ecological strategies that underlie microbial diversity remains limited [9-11].

Establishing the ecological principles that shape the distribution of microbial taxa is a major challenge [1214]. The importance of environmental factors on such distribution has been demonstrated, either considering

\footnotetext{
* Correspondence: jtamames@cnb.csic.es

Systems Biology Programme, Centro Nacional de Biotecnología (CNB-CSIC), C/Darwin 3, 28049 Madrid, Spain
}

(c) The Author(s). 2017 Open Access This article is distributed under the terms of the Creative Commons Attribution 4.0 International License (http://creativecommons.org/licenses/by/4.0/), which permits unrestricted use, distribution, and reproduction in any medium, provided you give appropriate credit to the original author(s) and the source, provide a link to the Creative Commons license, and indicate if changes were made. The Creative Commons Public Domain Dedication waiver (http://creativecommons.org/publicdomain/zero/1.0/) applies to the data made available in this article, unless otherwise stated.

only biotic factors $[13,14]$, or also including interactions among different taxa and abiotic factors [15]. Besides, the analysis of genomic data from an ecological point of view can provide a more comprehensive understanding of the structure and functioning of microbial communities $[16,17]$.

Several studies have analyzed the interrelationships among species considering the co-occurrences of different taxa in the same environments [12, 14, 18]. However, although thousands of fully sequenced genomes are available and some ecological traits have been investigated using metagenomic data $[11,15,19,20]$, these studies have mostly focused on specific environments. Recently, we have described the global relationships between genomic content, phylogenetic distance, and environmental preferences [21]. Following that approach, the main objective of this study is to find patterns between genomic characteristics of different prokaryotic 
taxa and both their ubiquity and their environmental preferences.

To this end, we addressed the relationships between these environment preferences and both genome size and the copy number of the 16S rRNA gene. We chose these genomic characteristics because previous studies related genome size to environmental versatility [22] and $16 \mathrm{~S}$ gene copy number to the potential growing rate [23]. In addition, we also analyzed the functional capabilities of each genus in comparison with its ecological strategies and environmental preferences. Since our study is focused on environmental versatility and growing rate, we will obtain insight on the ecological strategies to study linked to these traits, namely: (i) oligotrophic/copiotrophic microorganisms (growth rate) and (ii) generalist/specialist microorganisms (environmental versatility/ubiquity).

\section{Methods}

Genomic data were obtained for 2837 complete prokaryotic genomes with COGs (Clusters of Orthologous Groups) annotations [24, 25], taken from the National Center of Biotechnology Information (NCBI) [26]. As some genomes are scarcely annotated, we only considered these genomes in which the normalized ratio of annotated genes versus the total of genes fell within one standard deviation of the mean $(\bar{x}=71.5 \%, \mathrm{SD}=15.7)$. This resulted in 1420 genomes. We also calculated the genome size, the copy number of $16 \mathrm{~S}$ rRNA gene and the number of different copies of it (that is, non-identical 16S rRNA genes) for these genomes. Next, we created a functional profile of each genome by counting the abundance of individual COGs, that are classified in 18 individual functional classes. We normalized the number of ORFs (Open Reading Frames) and COGs in each functional class by dividing by the total number of ORFs and COGs, respectively. For validating purposes, we also created functional profiles based on KEGG (Kyoto Encyclopedia of Genes and Genomes) annotations [27].

We obtained the environmental data from the microDB data base (formerly envDB, http://botero.cnb.csic.es/envDB) [28], following the procedure in [29]. The database comprises more than 20,000 environmental samples and their associated 16S rRNA sequences, with each sample classified in a unique environment. By taxonomically classifying the $16 \mathrm{~S}$ sequences in these samples, it is possible to know which taxa are present in which samples and, consequently, in the corresponding environments. We chose the genus level as taxonomic working unit because it provides a good balance between a high number of taxa and not too sparse observations for them. Also, many $16 \mathrm{~S}$ sequences in microDB are partial and do not allow classification to ranks below genera.
In order to obtain the number of environments where each genus was found, we computed a table of the observed frequency of genera in environments (Additional file 1), excluding instances in which a genus was observed in just one sample of a particular environment. Using these frequencies, we tested the measure of the association genus-environment using Fisher's exact tests, adjusting the resulting $p$-values using the Benjamini and Hochberg FDR correction [30]. We considered that a taxon was linked to an environment when the adjusted $p$-value was lower than 0.01 . To assess that these significances were not random observations, we computed 100 random frequency tables and applied the same procedure to them. No significant associations were detected between genera and environments using these random matrices $(p$-value $=1)$. The number of genomes in each environment, and the number of environments linked to each genome, can be found in Additional file 2.

Since each genus can contain different species/strains, we averaged the values of the genome size, copy number of $16 \mathrm{~S}$ rRNA gene, the number of different $16 \mathrm{~S}$ rRNA genes and the COGs/KEGGs functional profile for the corresponding genomes.

Finally, to link COG presence with ubiquity, we calculated the average presence of each COG across all the genomes of a genus (thus obtaining a value between zero and one), and related that measure with the ubiquity of the genus.

All statistical tests were done using the software package R [31].

\section{Results}

Relationships of $16 \mathrm{~S}$ copy number, number of different $16 \mathrm{~S}$ genes, genome size and functional profile for every genus with ubiquity and environmental preferences

Figure 1 and Additional file 3 show the relationships between genome size, 16S rRNA copy number and number of different $16 \mathrm{~S}$ rDNA genes with the ubiquity of their corresponding genomes (Fig. 1a-c) and the particular environments associated with them (Fig. 1d-f, Additional file 3). Genome size increased with ubiquity (Fig. 1a), which was confirmed by a linear regression $\left(R^{2}=0.04\right.$, $p$-value $<0.01)$ and a Kruskal-Wallis significance test $(p$ value $<0.01$ ). In contrast, the $16 \mathrm{~S}$ rRNA gene copy number (Fig. 1b) did not vary according to the ubiquity (Kruskal-Wallis $p$-value $>0.01$ ). The number of different 16S rRNA genes did not vary either according to the ubiquity (Kruskal-Wallis $p$-value $>0.01$ ) (Fig. 1c).

Microorganisms living in different environments showed different trends regarding genome size (KruskalWallis $p$-value $<0.01)$ and $16 \mathrm{~S}$ rRNA copy number $(p$ value $<0.01$ ) (Fig. 1d-f). The genera occurring in hostassociated environments (gut and oral) showed both high copy number of $16 \mathrm{~S}$ rRNA genes and small genome 


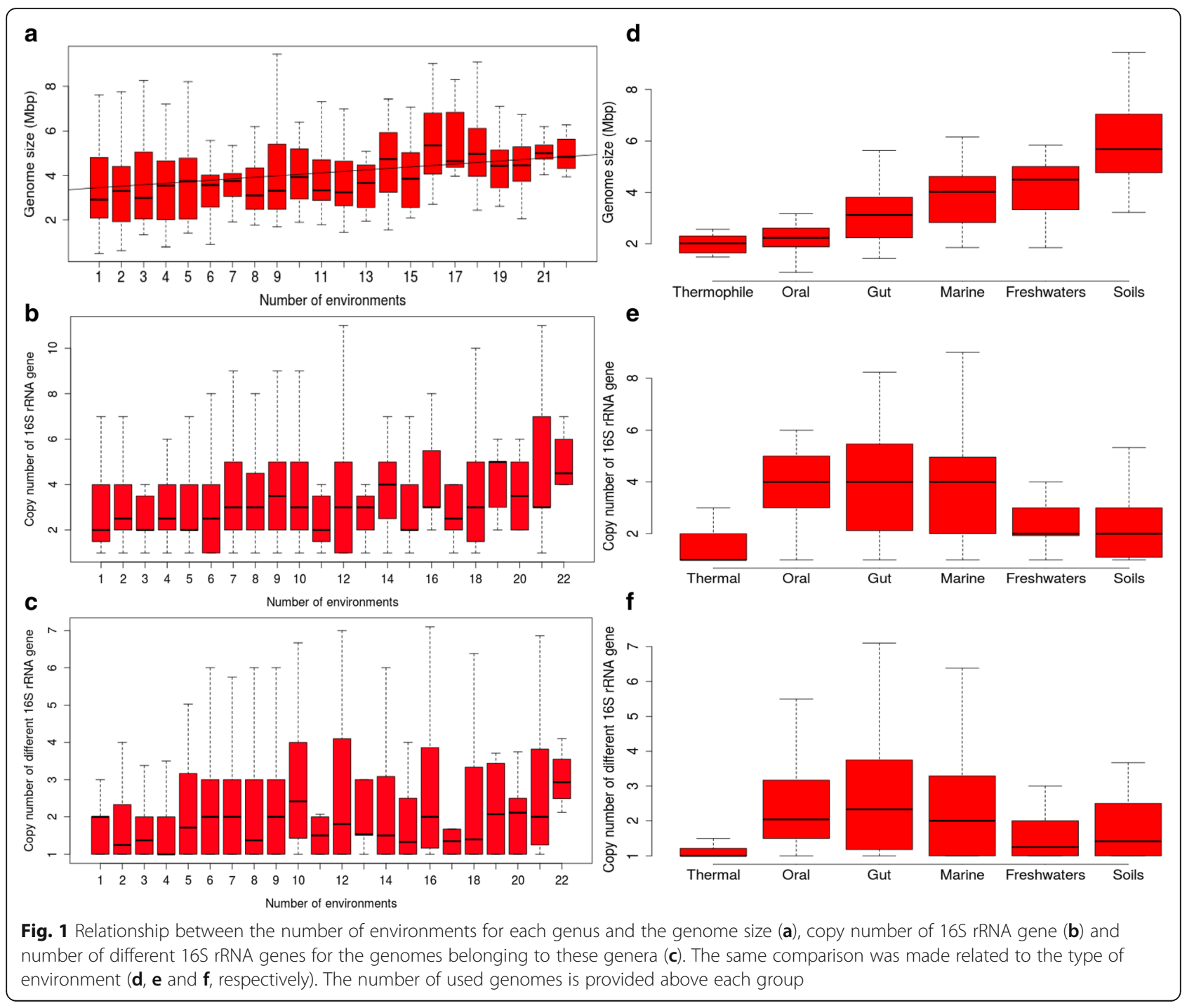

size (Fig. 1d-e, Additional file 3A). Thus, according to the results of Mann-Whitney statistical test, both environments did not present significant differences in copy number and genome size ( $p$-value $>0.01)$ but they were significantly different for genome size and copy number of $16 \mathrm{~S}$ rRNA gene from the rest of environments ( $p$ value $<0.01$ ) . Genera present in soils (Fig. 1d-e, Additional file 3B) showed significantly the largest genome sizes of the data set (Mann-Whitney $p$-value $<0.01$ ) but no statistical differences in 16S rRNA copy number with the other environments ( $p$-value $>0.01$ ) (Fig. 1d-e, Additional file 3C). Thermophilic bacteria inhabiting springs and submarine vents showed both significantly low $16 \mathrm{~S}$ rRNA copy number and small genome size (the smallest genomes) (Mann-Whitney test $p$-value <0.01) (Fig. 1d-e, Additional file 3D). Marine and freshwaters microorganisms did not present significant differences in genome size and 16S rRNA gene copy number (Additional file 3 ), but although both tended to have large genomes, freshwaters presented a slightly lower copy number of 16SrRNA gene than marine genera (Fig. 1d-e), being closer to soils microorganisms.. The results of the different $16 \mathrm{~S}$ rRNA genes showed the same tendency as $16 \mathrm{~S}$ copy number (Fig. 1c, f). According to these results, we can extract some general trends for environmental microorganisms, summarized in Fig. 2.

\section{Relationships between gene/functional class content, ubiquity and environmental preferences}

Next, we studied the distribution of functions in the genomes linked to the diverse environments, to gain insight on: 1) the adaptations to ubiquity, and 2) the adaptations to different environments.

We calculated the ratio of different COGs (Fig. 3a and b) and ORFs (Fig. 3c and d) that each genus devotes to each functional class, grouping the genera according to either the number of environments in which they were found (Fig. 3a and c), or by their preferred environments 


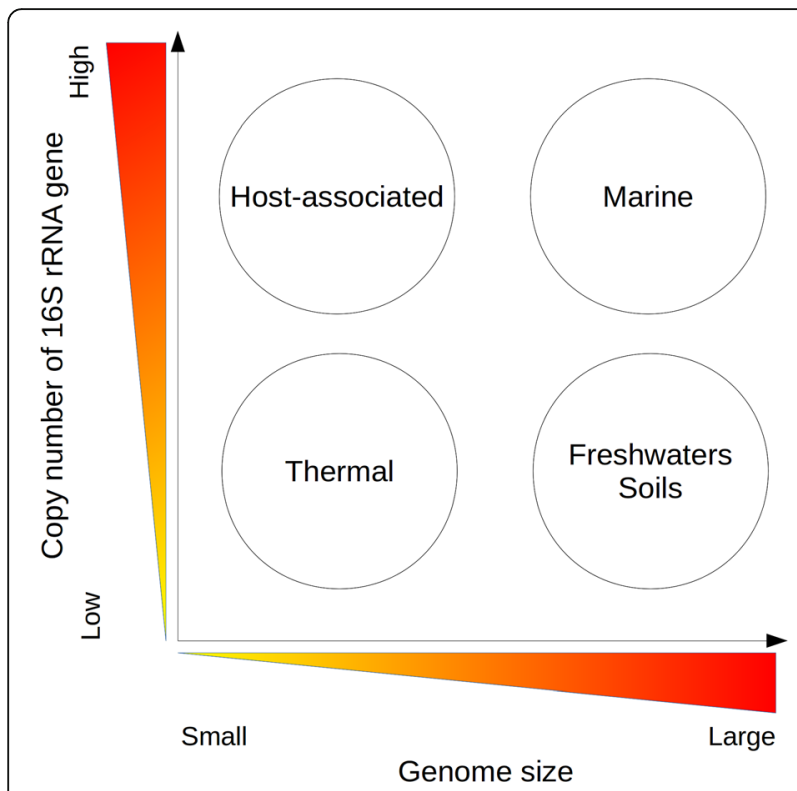

Fig. 2 Trends for genome size and copy number of the 16S rRNA gene in different environments

(Fig. 3b and d). The ratio of COGs will inform on the number of different protein families for a particular functional class, whereas the ratio of ORFs will tell us of the number of total genes for it.

To verify that these trends hold when using a different functional classification, we repeated the analyses using KEGG database (Additional file 4). The results indicate that equivalent functional classes follow the same trends almost entirely.

First we focused on studying the ratio of different COGs. For most functional classes, we did not find any correlation with the ubiquity of the corresponding bacteria (Fig. 3a). Only the classes of inorganic ion transport and metabolism and secondary metabolism increased their share in ubiquitous taxa ( $p$-value Kruskal-Wallis test $<0.01$, linear regression $p$-value $<0.01, R^{2}=0.61$ and 0.68 respectively). In contrast, translation, ribosomal structure and biogenesis significantly decreased with ubiquity ( $p$-value Kruskal-Wallis test $<0.01$, linear regression $p$-value $<0.01, \mathrm{R}^{2}=0.61$ ). When using the KEGG database, lipid metabolism, metabolism of other aminoacids and xenobiotics metabolism increased their ratio in ubiquitous taxa ( $p$-value Kruskal-Wallis test $<0.01, p$-value linear regression $<0.01, R^{2}=0.41,0.36$ and 0.50 respectively). On the other hand, folding, sorting and degradation; glycan biosynthesis and metabolism; nucleotide metabolism; transcription, and translation significantly decreased with ubiquity ( $p$-value Kruskal-Wallis test $<0.01$, linear regresion $p$-value $<0.01, \mathrm{R}^{2}=0.43,0.51,0.49,0.37$ and 0.68 respectively).

Most classes showed different abundances in different environments (Fig. 3b). Only two functional classes, defense mechanisms and coenzyme metabolism, were stable ( $p$-value Kruskal-Wallis test $>0.01)$. The rest showed clear differences.

We checked that these differences were not caused by different genomic sizes of the genomes associated to each environment, by fitting a linear regression of the ratio of COGs and ORFs in each functional class to the genomic size of the corresponding genomes. (Additional files 5 and 6 , respectively). Most regressions were not significant $(p<0.01)$ and therefore indicate that there is no relation between functional class abundance and genomic size in particular environments.

Regarding particular environments, host-associated habitats, such as oral and gut, were enriched in carbohydrate transport and metabolism. They also had high proportion of genes belonging to nucleotide metabolism, replication, transcription and translation, but this is probably linked to the smaller sizes of their genomes (Additional files 5 and 6). The same tendencies were observed using the KEGG database but adding a high proportion of genes devoted to membrane transport (Additional file 4). Microorganisms living in thermal environments have a high amount of genes devoted to energy metabolism, coenzyme metabolism, and posttranslational modification and chaperones. Concordantly, the same tendencies were observed in the KEGG database but adding a high proportion devoted to membrane transport in host-associated environments (Additional file 4). using KEGG database we also observe a high proportion of genes dedicated to vitamins and cofactors metabolism (Additional file 4B).

Soil microorganisms were enriched in carbohydrate metabolism, secondary metabolism, and were low in genes of the cell wall machinery and translation. In the COG database, they were also enriched in signal transduction systems (Fig. 3b).

Marine and freshwater organisms showed very similar profiles: they were enriched in motility, and secretion and signal transduction systems, while were depleted in carbohydrate metabolism (for freshwaters, this correlates with genomic size).

In concordance with previous studies, a larger percentage of cell motility genes was found in microorganisms living in open environments (thermal, marine [32], freshwaters [33] and soils [34-36]) than in hostassociated microorganisms (oral and gut).

When, instead of focusing on the ratio of COGs, we considered the ratio of ORFs (that is, the total number of genes for each class, Fig. 3c-d), the trends previously found not only hold, but are augmented.

Finally, we analyzed the relationship between the presence of some genes and the ubiquity of the corresponding genomes, to determine whether some genes promoted adaptation to the different and variable 


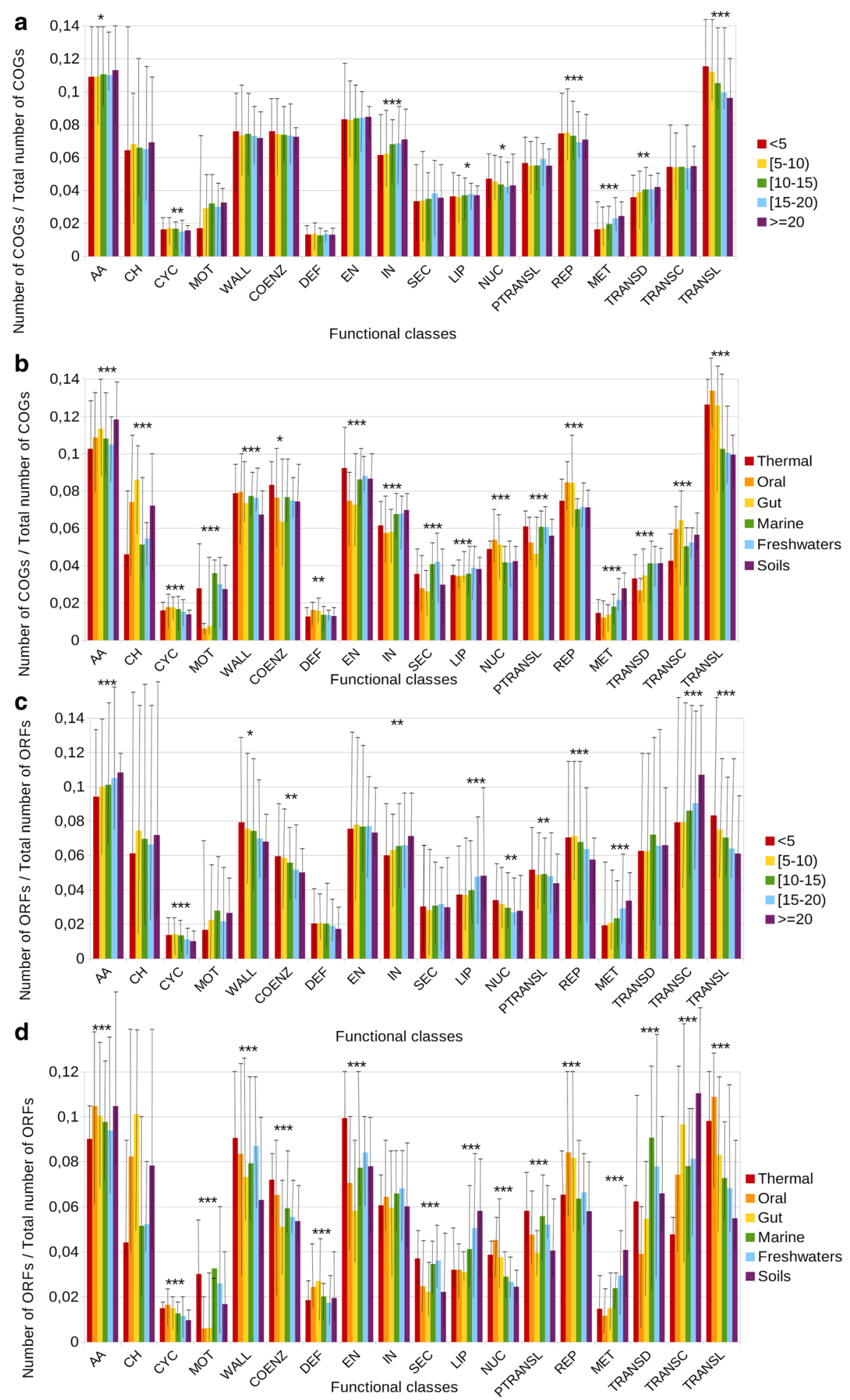

Fig. 3 Fraction of distinct COGs ( $\mathbf{a}$ and $\mathbf{b}$ ) and ORFs ( $\mathbf{c}$ and $\mathbf{d}$ ) dedicated to different functional classes regarding their ubiquity (a and $\mathbf{c}$ ), and the preferred environment for each genus ( $\mathbf{b}$ and $\mathbf{d}$ ). The error bars represent the range of the data 
conditions corresponding with diverse environments. To that end, we studied the distribution of the presence of each COG in organisms living in a given number of environments (pooling together the genera associated to that particular number of environments, and then calculating the average of the presence of each COG in the genomes of these genera). We used a linear regression between the COG presence ratio and the number of associated environments to study the presence of potential relationships between these two factors. We found that few, but some COGs were positively correlated ( $p$-value $\left.<0.01, \mathrm{R}^{2}>0.7\right)$ with the ubiquity of their corresponding taxa. (Table 1, Additional file 7).

\section{Discussion}

Relationships of $16 \mathrm{~S}$ copy number, number of different $16 \mathrm{~S}$ genes, genome size and functional profile with ubiquity and environmental preferences

The existence of a positive correlation between genomic size and ubiquity (Fig. 1), suggests that having a larger genome allow the microorganisms to be adapted to more environmental conditions, in concordance with previous studies [33, 37].

Also, since copy number of $16 \mathrm{~S}$ rRNA gene is likely related to fast growth potential [23, 38-45], the results indicated that this potential is uncorrelated with the adaptation to different environments. Similarly, although it has been suggested that microorganisms with multiple copies of the 16S rRNA gene can be more adaptable to changing environmental conditions and grow more readily on culture media $[23,46]$, we found that the presence of different $16 \mathrm{~S}$ rRNA genes did not correlate with ubiquity.

Alternative combinations of genome size and 16S rRNA copy number suggested diverse ecological strategies (growth rate and versatility, related to high copy number of 16S rRNA gene and large genome size, respectively [23]) for inhabiting different environments (Fig. 2).

The small genome size showed by host-associated (oral and gut) microorganisms is probably related to the stability of the environment, where fewer genes are required for adaptation. The extreme case is the genomic reduction experienced by symbionts and parasites $[45$, 47]. The high availability of nutrients may translate into an increased capacity for rapid growth [23], providing an explanation to their high $16 \mathrm{~S}$ rRNA copy number. Nevertheless, this copy number showed a high variability, suggesting that different growth strategies may be present in this environment.

Large genomes containing many genes, and consequently having increased metabolic potential, are probably more ecologically successful in environments where resources are scarce but diverse, and where there is little penalty for slow growth [48]. The probable low growth rate suggested by the low $16 \mathrm{~S}$ rRNA copy number in soil

Table 1 Genes whose abundance in genomes is linearly correlated to the ubiquity of these genomes

\begin{tabular}{|c|c|c|c|c|}
\hline Group & Functional class & Gene & COG & $\begin{array}{l}\text { Adjusted R- } \\
\text { squared }\end{array}$ \\
\hline \multirow[t]{3}{*}{$\begin{array}{l}\text { CELLULAR PROCESSES AND } \\
\text { SIGNALING }\end{array}$} & $\begin{array}{l}\text { Cell wall / membrane / envelope } \\
\text { biogenesis }\end{array}$ & Sortase and related acyltransferases & COG1247 & 0.7484 \\
\hline & $\begin{array}{l}\text { Posttranslational modification, protein } \\
\text { turnover, chaperones }\end{array}$ & Glutatione peroxidase & COG0386 & 0.8488 \\
\hline & Signal transduction mechanisms & $\begin{array}{l}\text { Osmosensitive } \mathrm{K}+ \\
\text { channel histidine kinase }\end{array}$ & COG2205 & 0.7662 \\
\hline \multirow[t]{3}{*}{$\begin{array}{l}\text { INFORMATION STORAGE AND } \\
\text { PROCESSING }\end{array}$} & Replication, recombination and repair & $\begin{array}{l}\text { Nucleotidyltransferase/DNA } \\
\text { polymerase involved in DNA repair }\end{array}$ & COG0389 & 0.73 \\
\hline & & A/G-specific DNA glycosylase & COG1194 & 0.7548 \\
\hline & $\begin{array}{l}\text { Translation, ribosomal structure and } \\
\text { biogenesis }\end{array}$ & $\begin{array}{l}\text { Acetyltransferases, including } \\
\mathrm{N} \text {-acetylases of ribosomal proteins }\end{array}$ & COG1670 & 0.7714 \\
\hline \multirow[t]{7}{*}{ METABOLISM } & Carbohydrate transport and metabolism & $\begin{array}{l}\text { 2,4-dihydroxyhept-2-ene-1,7-dioic } \\
\text { acid aldolase }\end{array}$ & COG3836 & 0.7147 \\
\hline & Coenzyme transport and metabolism & Dihydrofolate reductase & COG0262 & 0.8237 \\
\hline & Energy production and conversion & Glycerol-3-phosphate dehydrogenase & COG0578 & 0.7355 \\
\hline & & $\begin{array}{l}\text { Coenzyme F420-dependent N5, } \\
\text { N10-methylene tetrahydromethanopterin } \\
\text { reductase and related flavin-dependent } \\
\text { oxidoreductase }\end{array}$ & COG2141 & 0.7239 \\
\hline & Inorganic ion transport and metabolism & Phosphate/sulphate permeases & COG0306 & 0.7579 \\
\hline & Lipid transport and metabolism & $\begin{array}{l}\text { Acyl CoA: acetate/3-ketoacid CoA } \\
\text { transferase, alpha subunit }\end{array}$ & COG1788 & 0.7461 \\
\hline & Nucleotide transport and metabolism & Thymidilate synthase & COG0207 & 0.7334 \\
\hline
\end{tabular}


taxa (Fig. 1e) is supported by the abundance and dominance of slow growing oligotrophic $\alpha$-Proteobacteria, such as non-symbiotic member of the Rhizobiaceae and Bradyrhizobiaceae [23, 48-50] families. Generation times in soil are thought to be longer than other environments [40]. The presence of microenvironments and, therefore, the different growth strategies in soil environment, is reflected in the high dispersion observed in their 16S rRNA copy number (Additional file 3B). That is why the differences in 16S copy number between soils and the rest of the environments are not significant. The same explanation can be applied to freshwaters, that showed a similar trend, although with smaller genome sizes than in soils but not significantly different from the rest of the environments (Additional file 3C), suggesting a wide range of variation.

Genome size in thermophilic microorganisms may be an indirect target of selection due to its association with cell volume. Known changes in cell structure and physiology at high temperature can provide a selective advantage to reduce cell volume [51]. Also, the apparent stability of these environments can promote smaller genome sizes, as mentioned above. The low $16 \mathrm{~S}$ copy number can be explained by the stress conditions that penalize growth. In this regard, it is known that generation time increases when increasing habitat temperature [51].

Within the wide diversity of ecological strategies in seawater, oligotrophic bacteria are abundant [51]. These microbes grow slowly, in opposition to the fast-growing copiotrophs [52]. Despite their abundance, probably just a few taxa are oligotrophs, with small genomes and slow growth [53]. But most of the low-abundance taxa in the ocean could be capable of slow growth in energy-limited environments, and rapid growth in energy-rich environments, as suggested by their high copy number of $16 \mathrm{~S}$ rRNA [52]. Accordingly, high growth rates occur periodically in nutrient-rich microzones [53-55]. Thus, the slow average-growth rates that are typically observed for pelagic communities do not preclude the possibility that a small fraction of the assemblage might be growing rapidly [5658]. Copy numbers of the $16 \mathrm{~S}$ rRNA gene show a high variability, which can explain the diversity of ecological strategies due to the presence of pelagic microniches in seemingly homogeneous ocean waters [33, 59]. Thus, many genera showed both a high copy number of $16 \mathrm{~S}$ rRNA and a large genome, which may explain their capability of adapting to different situations. Both freshwaters and marine microorganisms presented a similar tendency in relation to $16 \mathrm{~S}$ rRNA gene copy number and genome size (wide range of values), reflected in the non-significant differences between them and the rest of microorganisms (Additional file $3 \mathrm{C}$ and $\mathrm{E}$ ).
Hence, we have found different combinations of genome size and 16S rRNA copy number that evidence different ecological strategies for inhabiting different environments (Fig. 2), supporting previous studies (soils, freshwaters and thermal environments $[48,51]$ ) or finding novel patterns for marine environments [51].

Finally, it has been proposed that a higher number of different 16S rRNA genes makes microorganisms more adaptable to variable conditions $[23,46]$, and therefore environments which display more stable conditions will harbor bacteria with fewer rRNA operons [60]. However, our results do not support this hypothesis (Fig. 1f): microorganisms related to environments with a high availability of nutrients (gut and oral) presented a high number of different $16 \mathrm{~S}$ rRNA genes, and also marine microorganisms.

\section{Relationships among gene/functional class content, ubiquity and environmental preferences}

In ubiquitous taxa, we observed an increase in the share of functional classes proposed to be more linked to environmental adaptation, such as inorganic ion transport and metabolism, secondary metabolism, xenobiotics biodegradation and metabolism and, to a lesser extent, signal transduction [21]. In contrast, the decrease of functions related to nucleotide metabolism, replication and translation in these ubiquitous genomes is significant and may be explained by the strong positive correlation between ubiquity and genome size. As the machinery related to nucleotide metabolism, replication and translation is well conserved for all organisms, the amount of genes for these functional classes is similar for all genomes, but the ratio is smaller in these with larger genomes.

The enrichment in carbohydrate transport and metabolism observed in host-associated genomes, such as oral and gut, is in agreement with the large amount of carbohydrates present in such environments [60, 61]. Organisms living in these environments also have high ratio of genes belonging to nucleotide metabolism, replication and translation because they tend to have small genomes. We observed the same trend for organisms living in thermal environments. These also have a high amount of genes devoted to energy metabolism, and to posttranslational modification and chaperones, probably to increase protein survival and maintenance. In this habitat, carbohydrate metabolism is minimal, probably a consequence of the autotrophic metabolism found in these environments.

The enrichment in carbohydrate metabolism in soil microorganisms may be explained by the different microenvironments found in soils, with many diverse nutrients and conditions [23, 62]. They are also enriched in secondary metabolism, which is probably related to the 
diverse set of potential nutrients available in the soils. Interestingly, they are short in genes of the cell wall machinery and translation. Both functional classes have been demonstrated to be functional indicators of copiotrophy [63], and their depletion is in concordance with the abundance and dominance of slow-growing oligotrophic $\alpha$-Proteobacteria.

Marine and freshwater organisms showed very similar profiles, as we could also see when studying 16S rRNA gene copy number and genome size, and it is tempting to hypothesize that they face similar environmental challenges. They are enriched in motility, and secretion and signal transduction systems, while depleted in carbohydrate metabolism, perhaps due to the abundance of autotrophic taxa in the photic zone, such as the phototroph Prochlorococcus. Also microorganisms in marine or freshwater sediments contribute to the anaerobic autotrophic metabolism.

When, instead of focusing on the number of different COGs/KEGGs, we studied the total number of ORFs belonging to these COGs/KEGGs (Fig. 3c-d and Additional file $4 \mathrm{C}-\mathrm{D}$ ), some interesting features that were obscured in the previous analysis emerged. For instance, while lipid metabolism was very stable when considering number of different COGs, there was a large increase in the number of genes related to this function in marine, freshwaters, and especially soil environments. That is, gene families in these classes have undergone expansion by duplication resulting in paralogs. Also there was a large increase in the transcription class in soils, because of the expansion of the COGs related to transcription factors. This is related to the creation of new regulatory proteins conferring increased fine-tuning of gene expression [64].

The negative linear correlation found between translation, ribosomal structure and biogenesis and genome size ( $p$-value $\left.<0.01, \mathrm{R}^{2}>0.7\right)$ for all environments is probably due to that the machinery needed for this process is highly conserved in all microorganisms, and therefore the ratio is smaller in bigger genomes.

Finally, ubiquity, and consequently adaptation, are promoted by strategies of pioneering, colonization, and protection from diverse stresses. This is noticeable by the positive relationship between ubiquity and the presence of genes like sortases and related acyltransferases, involved in cell wall/membrane/envelope biogenesis, strategies for bacterial escape from the host's immune response and creation of biofilms [65], that can promote colonization processes. Many genes related to protection from diverse stresses were also found: signal transduction proteins such as the osmosensitive $k+$ channel histidine kinase, which has an important role in osmoregulation and the chemotaxis system, participating in control complex processes such as the initiation of development in microorganisms; histidine kinase twocomponent systems, that are extremely common in bacteria and play an important role in signal transduction that is essential for adaptation to bacterial stress [66]; gluthatione peroxidase, whose main biological role is to protect the organism from oxidative damage, related to posttranslational modification protein turnover chaperones [67]. And finally, nucleotidyltransferase/DNA polymerase and A/G-specific DNA glycosylases, both involved in DNA repair $[68,69]$.

\section{Conclusions}

The study of the relationships between 16S copy number, genome size and adaptation to diverse environments showed that adaptation was linked to genome size $[37,38]$. In contrast, $16 \mathrm{~S}$ copy number was not directly related to ubiquity, highlighting that growth potential (related to $16 \mathrm{~S}$ copy number [23, 40-46]) does not favor the ubiquity. We observed distinctive trends regarding these two features: Small genome size and low $16 \mathrm{~S}$ copy number, found in thermal environments. This was probably the result of a direct targeting of natural selection on cell size, because their metabolic analysis suggested that large cells could suffer significant fitness costs at high temperatures [51]. Low genome size and high 16S copy number was found in host-associated habitats, as a result of the stability of the environment and the potential high growth rate of the organisms in them.

Larger genome sizes were found in environmental organisms from soils, freshwaters and the sea. The first two had a low $16 \mathrm{~S}$ copy number. This is in accordance with complex environments with many diverse niches, where organisms tend to be slow growers. Large genomes are supposed to be more ecologically successful in environments where resources are scarce but diverse and where there is little penalty for slow growth [48].

Finally, large genomes with high $16 \mathrm{~S}$ copy number were found in marine environments. This is probably related to the capacity of marine microorganisms for rapid growth in appropriate conditions. We hypothesize that, although most of marine microorganisms are oligotrophs, they consist on only a few taxa. In contrast, the majority of the taxa in the sea may be, in fact, copiotrophs, increasing their growth when a higher quantity of nutrients is available.

However, although marine microorganisms tend to have higher copy number of 16S rRNA gene than soils and freshwaters, these differences were not significant (Additional file 3), as well as the differences in genome size of freshwater and marine microorganisms, suggesting a wide range of variation in both genome size and $16 \mathrm{~S}$ copy number explained by the presence of microniches in these three environments. 
The analysis of functional classes showed some clear signatures in different environments, as described previously [21]. For example, a higher presence of carbohydrate metabolism and defense mechanisms in gut and oral cavity, reflecting the availability of nutrients and the need of defense mechanisms against the host immune system, and the fact that mobility may not be necessary in these environments. Another example is the high fraction of COGs devoted to nucleic acid functions in thermophilic microorganisms, which could be due to the need of frequent repair of DNA damage caused by the extreme conditions. Regarding the number of environments in which the organisms were able to live in, there were almost no differences between functional classes. Only few classes were correlated with the adaptation to a high number of environments: inorganic ion transport and metabolism, secondary metabolism, and signal transduction.

\section{Additional files}

Additional file 1: Procedure for obtaining associations between taxa and environments. Only the genera significantly related to some environment are shown in the Fisher's exact test table. ID. (PDF $18 \mathrm{~kb}$ )

Additional file 2: Number of genomes associated to each environment and to each number of environments. (XLSX $5 \mathrm{~kb}$ )

Additional file 3: Plots relating the average genome size of every genus to their average number of $16 \mathrm{~S}$ rRNA genes. All genera are shown, highlighting these associated to particular environments: (A) Hostassociated (B) Soil samples. (C) Freshwater samples. (D) Thermal samples (E) Marine samples (.pdf format). The $p$-values of the Mann-Whitney test applied to the genome size and 165 rRNA gene copy number between the genera associated to a particular environment and the rest are provided in the figures. (PDF $758 \mathrm{~kb}$ )

Additional file 4: Fraction of distinct KEGGs (A and B) and ORFs (C and D) dedicated to different functional classes regarding their ubiquity (A and $C$ ), and the preferred environment for each genus ( $B$ and $D$ ). The error bars represent the range of the data. (PDF $118 \mathrm{~kb}$ )

Additional file 5: Linear regressions between COG ratios and genome size, for genera with different environmental preferences. Only significant instances ( $p$-value $<0.01$ ) are shown. (PDF $199 \mathrm{~kb}$ )

Additional file 6: Linear regressions between ORF ratio and genome size for genera with different environmental preferences. Only significant instances ( $p$-value $<0.01$ ) are shown. (PDF $176 \mathrm{~kb}$ )

Additional file 7: Linear regression between the number of environments associated to the genera, and the average presence of some COGs in these genera, showing linear relationships between presence of some genes and the ubiquity of the corresponding genomes (.pdf format) (A) Sortase and related acyltransferases (B) Glutatione peroxidase. (C) Osmosensitive $\mathrm{K}+$ channel histidine kinase (D) Nucleotidyltransferase/DNA polymerase involved in DNA repair (E) A/Gspecific DNA glycosylase (F) Acetyltransferases, including $\mathrm{N}$-acetylases of ribosomal proteins (G) 2,4-dihydroxyhept-2-ene-1,7-dioic acid aldolase (H) Dihydrofolate reductase (I) Glycerol-3-phosphate dehydrogenase (J) Coenzyme F420-dependent N5,N10-methylene tetrahydromethanopterin reductase and related flavin-dependent oxidoreductase (K) Phosphate/ sulphate permeases (L) Acyl CoA: acetate/3-ketoacid CoA transferase, alpha subunit (M) Thymidilate synthase. (PDF $175 \mathrm{~kb}$ )

\section{Abbreviations}

COG: Clusters of Orthologous Groups; KEGG: Kyoto enciclopedia of genes and genomes; NCBI: National center for biotechlology information; ORF: Open reading frame

\section{Acknowledgments}

We would like to thank Carlos Pedrós-Alió for assistance in reviewing this article.

\section{Funding}

This work was supported by the project CTM2013-48292-C3-2-R (MINECO/ FEDER), Spain. MCS is supported by research scholarship BES-2014-070375 (MINECO), Spain. The funding bodies had no role in the design of the study, writing the manuscript and the collection, analysis, and interpretation of data.

\section{Availability of data and materials}

The data sets used and/or analyzed during the current study are available from the following databases:

Genome data: complete genomes from NCBI database http://

www.ncbi.nlm.nih.gov/genomes//proks.cgi. No permissions required. Publicly accessible.

Environmental and taxonomic information: microDB database (extracted in 12th of April 2015) http://botero.cnb.csic.es/envDB. No permissions required. Publicly accessible.

Functional assignment of different genes:

COG database: http://www.ncbi.nlm.nih.gov/COG/. No permissions required. Publicly accessible.

KEGG database: http://www.genome.jp/kegg/. No permissions required. Restricted access.

\section{Authors' contributions}

JT conceived and designed the analysis. MCS analysed and interpreted the data. Both authors wrote the paper, read and approved the final manuscript.

Ethics approval and consent to participate

Not applicable.

\section{Consent for publication}

Not applicable.

\section{Competing interests}

The authors declare that they have no competing interests.

\section{Publisher's Note}

Springer Nature remains neutral with regard to jurisdictional claims in published maps and institutional affiliations.

Received: 20 February 2017 Accepted: 21 June 2017

Published online: 29 June 2017

\section{References}

1. Janssen PH, Yates PS, Grinton BE, Taylor PM, Sait M. Improved culturability of soil bacteria and isolation in pure culture of novel members of the divisions Acidobacteria, Actinobacteria, Proteobacteria, and Verrucomicrobia. Appl Environ Microbiol. 2002;68:2391-6.

2. Joseph SJ, Hugenholtz P, Sangwan P, Osborne CA, Janssen PH. Laboratory cultivation of widespread and previously uncultured soil bacteria. Appl Environ Microbiol. 2003;69:7210-5.

3. Button DK, Schut F, Quang P, Martin R, Robertson BR. Viability and isolation of marine bacteria by dilution culture: theory, procedures, and initial results. Appl Environ Microbiol. 1993;59:881-91.

4. Connon SA, Giovannoni SJ. High-throughput methods for culturing microorganisms in very-low-nutrient media yield diverse new marine isolates. Appl Environ Microbiol. 2002;68:3878-85.

5. Rappé MS, Connon S a, Vergin KL, Giovannoni SJ. Cultivation of the ubiquitous SAR11 marine bacterioplankton clade. Nature. 2002;418:630-3.

6. Cho JC, Vergin KL, Morris RM, Giovannoni SJ. Lentisphaera araneosa gen. nov., sp. nov, a transparent exopolymer producing marine bacterium, and the description of a novel bacterial phylum, Lentisphaerae. Environ Microbiol. 2004;6:611-21.

7. Zengler K, Toledo G, Rappe M, Elkins J, Mathur EJ, Short JM, et al. Cultivating the uncultured. Proc Natl Acad Sci U S A. 2002;99:15681-6.

8. Handelsman J. Metagenomics: application of genomics to uncultured microorganisms. Microbiol Mol Biol Rev. 2004;68:669-85. 
9. Raes J, Foerstner KU, Bork P. Get the most out of your metagenome: computational analysis of environmental sequence data. Curr Opin Microbiol. 2007;10:490-8

10. Kunin V, Raes J, Harris JK, Spear JR, Walker JJ, Ivanova N, et al. Millimeterscale genetic gradients and community-level molecular convergence in a hypersaline microbial mat. Mol Syst Biol. 2008;4:198.

11. Raes J, Letunic I, Yamada T, Jensen LJ, Bork P. Toward molecular trait-based ecology through integration of biogeochemical, geographical and metagenomic data. Mol Syst Biol. 2011;7:473.

12. Freilich S, Zarecki R, Eilam O, Segal ES, Henry CS, Kupiec M, et al. Competitive and cooperative metabolic interactions in bacterial communities. Nat Commun. 2011;2:589.

13. Chaffron S, Rehrauer H, Pernthaler J, Von Mering C. A global network of coexisting microbes from environmental and whole-genome sequence data. Genome Res. 2010;20:947-59.

14. Freilich S, Kreimer A, Meilijson I, Gophna U, Sharan R, Ruppin E. The largescale organization of the bacterial network of ecological co-occurrence interactions. Nucleic Acids Res. 2010;38:3857-68.

15. Williams RJ, Howe A, Hofmockel KS. Demonstrating microbial co-occurrence pattern analyses within and between ecosystems. Front Microbiol. 2014;5:358.

16. Green JL, Bohannan BJM, Whitaker RJ. Microbial biogeography: from taxonomy to traits. Sci. 2008:320:1039-43.

17. Prosser Jl, Bohannan BJM, Curtis TP, Ellis RJ, Firestone MK, Freckleton RP, et al. The role of ecological theory in microbial ecology. Nat Rev Microbiol. 2007:5:384-92

18. Tamames J, Abellán JJ, Pignatelli M, Camacho A, Moya A. Environmental distribution of prokaryotic taxa. BMC Microbiol. 2010;10:85.

19. Barberán A, Fernández-Guerra A, Bohannan BJM, Casamayor EO. Exploration of community traits as ecological markers in microbial metagenomes. Mol Ecol. 2012;21:1909-17.

20. Ugalde JA, Gallardo MJ, Belmar C, Muñoz P, Ruiz-Tagle N, Ferrada-Fuentes S, et al. Microbial life in a fjord: metagenomic analysis of a microbial mat in Chilean Patagonia. PLoS One. 2013;8:e71952.

21. Tamames J, Sánchez PD, Nikel PI, Pedrós-Alió C. Quantifying the relative importance of phylogeny and environmental preferences as drivers of gene content in prokaryotic microorganisms. Front Microbiol. 2016;7:433.

22. Litchman E. Invisible invaders: non-pathogenic invasive microbes in aquatic and terrestrial ecosystems. Ecol Lett. 2010;13(12):1560-72.

23. Klappenbach J a, Dunbar JM, Schmidt TM. rRNA operon copy number reflects ecological strategies of bacteria. Appl Environ Microbiol. 2000;66: 1328-33.

24. COG database. National Center of Biotechnology information. Rockville Pike, Bethesda: US National Library of Medicine; 1997. http://www.ncbi.nlm.nih. gov/COG/

25. Tatusov RL, Galperin MY, Natale DA, Koonin EV. The COG database: a tool for genome-scale analysis of protein functions and evolution. Nucleic Acids Res. 2000;28:33-6.

26. National Center of Biotechnology Information. Rockville Pike, Bethesda: US National Library of Medicine; 1988. http://www.ncbi.nlm.nih.gov/genomes/ Iproks.cgi . Accessed Nov 1988.

27. Kanehisa M, Susumu G. KEGG: kyoto encyclopedia of genes and genomes. Nucleic Acids Res. 2000;28(1):27-30.

28. microDB database. Madrid: Biotechnology National Center; 2009. http:// botero.cnb.csic.es/envDB . Accession by Javier Tamames. 29 April 2009.

29. Pignatelli M, Moya A, Tamames J. EnvDB, a database for describing the environmental distribution of prokaryotic taxa. Environ Microbiol Rep. 2009;1:191-7.

30. Benjamini $Y$, Hochberg Y. Controlling the false discovery rate: a practical and powerful approach to multiple testing. J R Stat Soc B. 1995;57:289-300.

31. R. The R project for statistical computing. http://www.r-project.org Accessed Aug 1993.

32. Azam F, Malfatti F. Microbial structuring of marine ecosystems. Nat Rev Microbiol. 2007;5:782-91.

33. Matz C, Jürgens K. High motility reduces grazing mortality of planktonic bacteria. Appl Environ Microbiol. 2005;71:921-9.

34. Turnbull G a, JAW M, Whipps JM, Saunders JR. The role of motility in the in vitro attachment of Pseudomonas putida PaW8 to wheat roots. FEMS Microbiol Ecol. 2001;35:57-65.

35. De WS, Vermeiren H, IHM M, Kuiper I, Hendrickx N, Guido V, et al. Flagelladriven Chemotaxis towards Exudate components is an important trait for tomato root colonization by Pseudomonas fluorescens. Mol Plant-Microbe Interact. 2002;15:1173-80.
36. Capdevila S, Martínez-Granero FM, Sánchez-Contreras M, Rivilla R, Martín M. Analysis of Pseudomonas fluorescens F113 genes implicated in flagellar filament synthesis and their role in competitive root colonization. Microbiology. 2004;150:3889-97.

37. Bentkowski P, Van Oosterhout C, Mock T. A model of genome size evolution for prokaryotes in stable and fluctuating environments. Genome Biol Evol. 2015;7:2344-51.

38. Parter M, Kashtan N, Alon U. Environmental variability and modularity of bacterial metabolic networks. BMC Evol Biol. 2007;7:169.

39. Case RJ, Boucher Y, Dahllöf I, Holmström C, Doolittle WF, Kjelleberg S. Use of 165 rRNA and rpoB genes as molecular markers for microbial ecology studies. Appl Environ Microbiol. 2007;73:278-88.

40. Stevenson BS, Schmidt TM. Life history implications of rRNA gene copy number in Escherichia coli. Society. 2004;70:6670-7.

41. Blazewicz SJ, Barnard RL, Daly RA, Firestone MK. Evaluating rRNA as an indicator of microbial activity in environmental communities: limitations and uses. ISME J. 2013;7:2061-8.

42. Lauro FM, McDougald D, Thomas T, Williams TJ, Egan S, Rice S, et al. The genomic basis of trophic strategy in marine bacteria. Proc Natl Acad Sci U S A. 2009;106:15527-33.

43. Aiyar SE, Gaal T, Gourse RL. rRNA promoter activity in the fast-growing bacterium Vibrio natriegens. J Bacteriol. 2002;184:1349-58.

44. Shrestha PM, Noll M, Liesack W. Phylogenetic identity, growth-response time and rRNA operon copy number of soil bacteria indicate different stages of community succession. Environ Microbiol. 2007:9:2464-74.

45. Vieira-Silva S, Rocha EPC. The systemic imprint of growth and its uses in ecological (meta) genomics. PLoS Genet. 2010;6:e1000808.

46. Condon C, Squires C, Squires CL. Control of rRNA transcription in Escherichia coli. Microbiol Rev. 1995;59(4):623-45.

47. Moran NA, Wernegreen JJ. Lifestyle evolution in symbiotic bacteria: insights from genomics. Trends Ecol Evol. 2000;15:321-6.

48. Konstantinidis KT, Tiedje JM. Trends between gene content and genome size in prokaryotic species with larger genomes. Proc Natl Acad Sci U S A. 2004;101:3160-5.

49. Mitsui H, Gorlach K, Lee HJ, Hattori R, Hattori T. Incubation time and media requirements of culturable bacteria from different phylogenetic groups. J Microbiol Methods. 1997:30:103-10.

50. Saito A, Mitsui H, Hattori R, Minamisawa K, Hattori T. Slow-growing and oligotrophic soil bacteria phylogenetically close to Bradyrhizobium japonicum. FEMS Microbiol Ecol. 1998;25:277-86.

51. Sabath N, Ferrada E, Barve A, Wagner A. Growth temperature and genome size in bacteria are negatively correlated, suggesting genomic streamlining during thermal adaptation. Genome Biol Evol. 2013;5:966-77.

52. Yooseph $\mathrm{S}$, Nealson KH, Rusch DB, McCrow JP, Dupont CL, Kim M, et al. Genomic and functional adaptation in surface ocean planktonic prokaryotes. Nature. 2010;468:60-6.

53. Schlegel HG, Zaborosch C, Kogut M. General microbiology. 7th edition. Cambridge: Cambridge University Press; 1993.

54. Mouriño-Pérez RR, Worden AZ, Azam F. Growth of Vibrio cholerae 01 in red tide waters off California. Appl Environ Microbiol. 2003;69:6923-31.

55. Worden AZ, Seidel M, Smriga S, Wick A, Malfatti F, Bartlett D, et al. Trophic regulation of Vibrio cholerae in coastal marine waters. Environ Microbiol. 2006:8:21-9.

56. Hamasaki K, Long RA, Azam F. Individual cell growth rates of marine bacteria, measured by bromodeoxyuridine incorporation. Aquat Microb Ecol. 2004;35:217-27.

57. Rodriguez GG, Phipps D, Ishiguro K, Ridgway HF. Use of a fluorescent redox probe for direct visualization of actively respiring bacteria. Appl Environ Microbiol. 1992:58:1801-8.

58. Lebaron P, Servais $P$, Agogué $H$, Courties C, Joux F. Does the high nucleic acid content of individual bacterial cells allow us to discriminate between active cells and inactive cells in aquatic systems? Appl Environ Microbiol. 2001;67:1775-82.

59. Gasol JM, Li Zweifel U, Peters F, Fuhrman JA, Hagström Å. Significance of size and nucleic acid content heterogeneity as measured by flow cytometry in natural planktonic bacteria. Appl Environ Microbiol. 1999:65:4475-83.

60. Acinas SG, Klepac-Ceraj V, Hunt DE, Pharino C, Ceraj I, Distel DL, et al. Finescale phylogenetic architecture of a complex bacterial community. Nature. 2004:430:551-4 
61. Acinas SG, Marcelino LA, Klepac-Ceraj V, Polz MF. Divergence and redundancy of $16 \mathrm{~S}$ rRNA sequences in genomes with multiple rrn Operons. J Bacteriol. 2004;186:2629-35.

62. Young IM, Crawford JW. Interactions and self-organization in the soilmicrobe complex. Science. 2004;304:1634-7.

63. Foster RC. Microenvironments of soil microorganisms. Biol Fertil Soils. 1988; 6:189-203.

64. Bastida F, Selevsek N, Torres IF, Hernández T, García C. Soil restoration with organic amendments: linking cellular functionality and ecosystem processes. Sci Rep. 2015;5:15550. BMC Syst Biol 2013, 7:127

65. Spirig T, Weiner EM, Clubb RT. Sortase enzymes in gram-positive bacteria. Mol Microbiol. 2011;82:1044-59.

66. Matsushita M, Janda KD. Histidine kinases as targets for new antimicrobial agents. Bioorg Med Chem. 2002;10:855-67.

67. Espinoza SE, Guo H, Fedarko N, DeZern A, Fried LP, Xue Q-L, et al. Glutathione peroxidase enzyme activity in aging. J Gerontol A Biol Sci Med Sci. 2008;63:505-9.

68. Dufresne A, Garczarek L, Partensky F. Accelerated evolution associated with genome reduction in a free-living prokaryote. Genome Biol. 2005;6:R14.

69. Aravind L, Koonin EV. DNA polymerase beta-like nucleotidyltransferase superfamily: identification of three new families, classification and evolutionary history. Nucleic Acids Res. 1999;27:1609-18.

Submit your next manuscript to BioMed Central and we will help you at every step:

- We accept pre-submission inquiries

- Our selector tool helps you to find the most relevant journal

- We provide round the clock customer support

- Convenient online submission

- Thorough peer review

- Inclusion in PubMed and all major indexing services

- Maximum visibility for your research

Submit your manuscript at www.biomedcentral.com/submit
Biomed Central 